\title{
Usage of the game technologies engines for the purpose of modern geographic information systems creation
}

\author{
Kirill Zhigalov ${ }^{1,2, a}$ \\ ${ }^{1}$ V.A. Trapeznikov Institute of Control Sciences of Russian Academy of Sciences, 117997 Moscow, Russia \\ ${ }^{2}$ Moscow Technological Institute, 119334 Moscow, Russia
}

\begin{abstract}
Modern professional geographic information systems significantly lagged behind in quality of 3D graphics, which complicates their full application in the existing conditions. The main differences of the principles of game engines work from the principles of creation of professional geographic information systems structure are considered in the article. These aspects interfere with construction of modern from the graphic point of view, geographic information systems. The analysis of the contradictions considered in article will allow minimizing them at application of game engines (for example, Unity) for reduction in cost and acceleration of production of modern GIS of new generation.
\end{abstract}

\section{Introduction}

The three-dimensional graphics allows presenting better various processes and the phenomena to people without vocational education and spatial imagination. Unfortunately, modern geographic information systems were developed more than 10 years ago. At that time, the most part of loading on reproduction of graphics on monitors laid down on the system processor but not on the graphic processors. It causes their essential lag in a graphic component expressed not only in slow display of a scene, but also in impossibility to reproduce modern graphic effects (for example, transparency).

In a situation with games everything is absolutely opposite: they are constructed on all modern technologies and allow using all resources of the modern computer as much as possible. As, the majority of them are constructed on the unified graphic engines, such as: Unity, Unreal Development kit, Cry engine would, etc. logical to use them for fast creation of the modern geoinformation supplement. Nevertheless, there is some of obstacles to this decision connected with initial orientation of graphic engine to computer games:

- Only the relative system of coordinates, with the center in a graphic scene is used. While standard GIS use generally geographical systems of coordinates;

- The seamless repeating textures at most in HD permission $(1920 * 1080$ pixels) are used. GIS uses aero photo filming, space pictures, etc. They don't repeat and can have permission in some tens of thousands of pixels on each of the parties;

- Objects have to contain the minimum quantity of the triangles (grounds) in a scene, and the maximum quality of the picture is reached by textures. GIS aspires to the

\footnotetext{
a Corresponding author: kshakalov@mail.ru
}

accuracy of mathematical models, respectively strives for the maximum number of triangles;

- Positioning of objects in a scene is carried out "approximately". It is necessary in GIS to have the exact geographic position of objects (with an accuracy of centimeters);

- There is no possibility to display the cuts of the objects, which are cut off by other objects.

\section{Use only of relative system of coordinates}

For game system and also 3D systems of modeling (3DsMAX, for example) there is no need to use difficult system of coordinates, such as the relative system of coordinates. Meanwhile all GIS programs use geographical systems of coordinates.

It looks naturally to use recalculation of coordinates for display them on the screen, input-output of data and their adjustment in such systems. At such implementation of a question the scene is building concerning the Center of the world - a reference point, from which all others will be shifted, at this parameters:

- Latitude;

- Longitude;

- Exceeding.

The software has to carry out transformation from relative system of coordinates to geographical angular or metric. Thus the internal system of coordinates remains in meters with the center in $0,0,0$. The main transformations can be made in a code rather compactly and precisely through mathematics of quaternions. For recalculation between various systems of coordinates can be used Russian standard GOST P 51794-2008 [1]. In 
more detail about it is possible to see formulas in it. It is possible to see an equivalent code in figure 1.

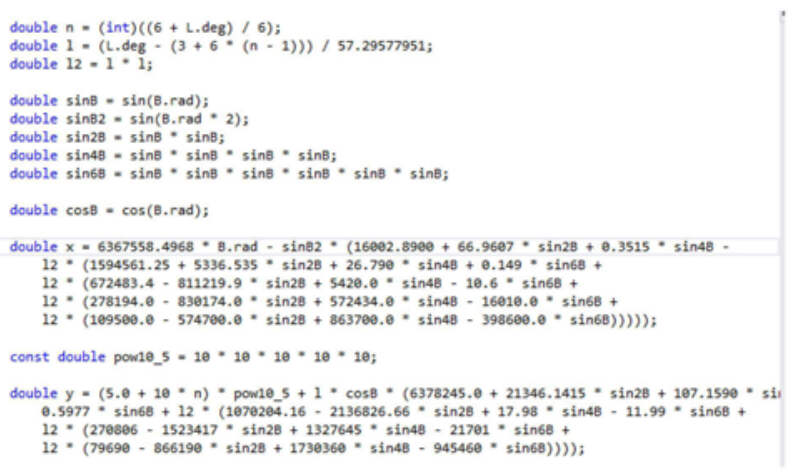

Figure 1. A program code fragment on $\mathrm{C}++$, responsible for recalculation of systems of coordinates.

Recalculation accuracy on a test example was checked by converting data between angular system of coordinates, system of coordinates of SK-42 (which it is still rather widespread in Russia Federation) and local system of coordinates of the program. Accuracy of converting reached $100 \%$

\section{Use of the seamless repeating textures}

In games there is no need to display the district precisely since it isn't real model. In geographic information systems the district has to be displayed rather precisely in this connection, the repeating seamless textures can't be applied.

The only way out is the greatest possible compression of entrance data (for example, photos) in graphic editors before you can see emergence of visual distortions. Usually, the standard image compresses to $70 \%$ which significantly increases software productivity.

\section{Minimum quantity of triangles (planes) in models}

In the most part of games, constructions and a parts of relief is replaced in a special way the made textures. GIS strives for the greatest possible geometrical compliance of each object. Nevertheless, amount of objects in a scene of GIS system can reach several hundred, and at the maximum compliance of each object to its real geometrical parameters, the number of at the triangles, displayed in the same time, will aspire to several million. Any modern graphic platform isn't able to make such quantity calculations. As a result, the task is reduced to a certain compromise.

For this purpose, during $3 \mathrm{~d}$ modeling of objects for GIS it is necessary to choose parameters, important for the specific project, and apply only them. As shown in Figure 2 - fragments of ladders, lampposts and metalwork are simulated, and windows and walls are made in the form of textures on an equal surface.

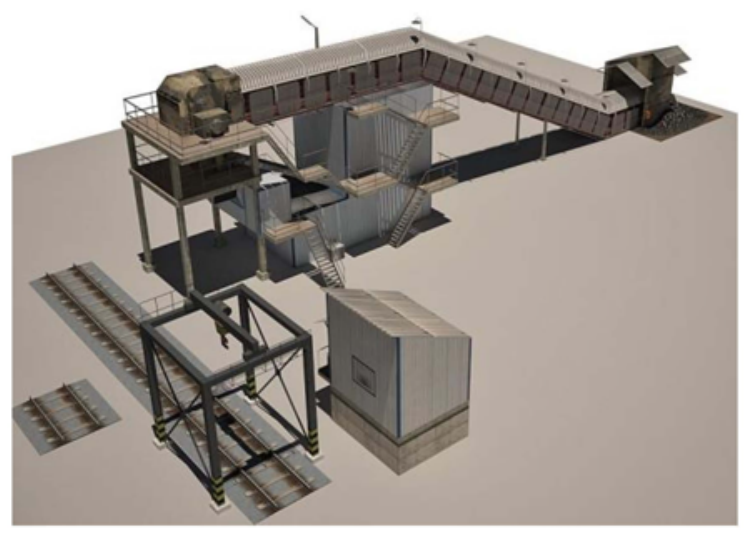

Figure 2. Example of production of model in the hybrid way.

\section{Positioning of objects in a scene}

In game systems 2 types of positioning are used:

- approximately;

- on two points.

Unfortunately, both types of positioning of objects aren't suitable for GIS of systems. It is connected with the insufficient accuracy of their placement at such approach. In geographic information systems the following approaches are applied:

- by the three points (characteristic points on available a relief or a scene get out and are compared with the corresponding or adjoining points on the positioned model);

- by coordinates (matching on model not less than 3 points in the system of coordinates which is used in GIS, then the model at its loading is automatically positioned in a geographic information system).

Respectively, it is necessary to make updates to a graphic engine for possibility of such actions. They will be enough, for receiving the qualitative, measured and detailed visual images.

\section{Lack of possibility of display of cuts of the objects which are cut off by other objects}

In games everything is presented in the form of models and cuts aren't required. In the geographic information systems focused on a markscheidery, for example, cuts are simply necessary for obtaining visual information on layers of rocks (see figure 3).

At creation of difficult systems of display, such as the pit surface with simultaneous display of layers, at us already turns out a cut of the object which is cut off by other objects (see figures 4,5 ).

For such displays it is expedient to use the passes of a render involved for the minor purposes in games (for example: depths of color, or refraction). This method has also a reverse side - absence of these effects at the time of should be noted that at the time of writing of article there was a question of display of cut of layers in places 
of their crossing with the relief existing for other date. The question remains open.

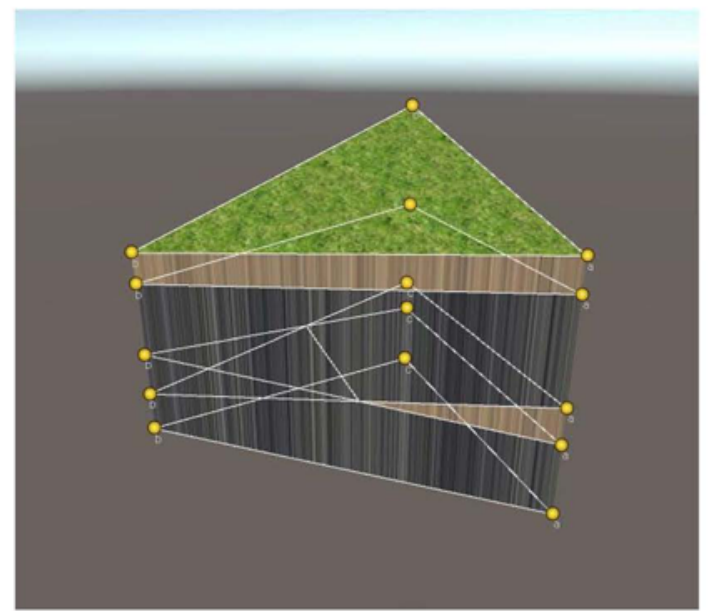

Figure 3. An example of visualization of rocks with use of cuts.

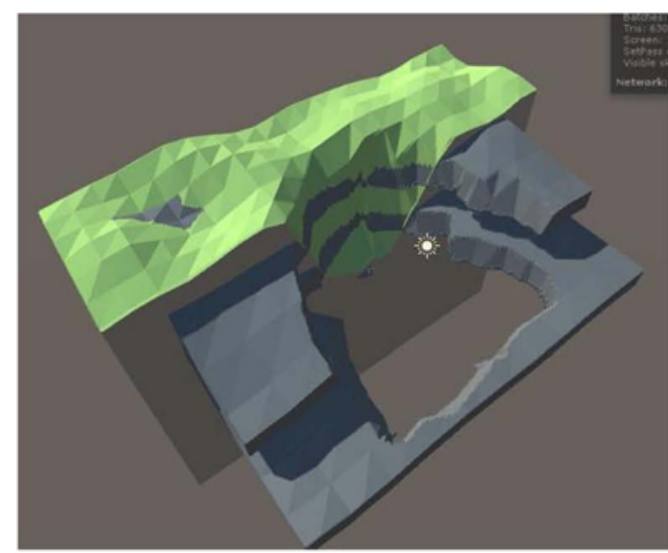

Figure 4. An example of display of a cut of the object which is cut off by other objects.

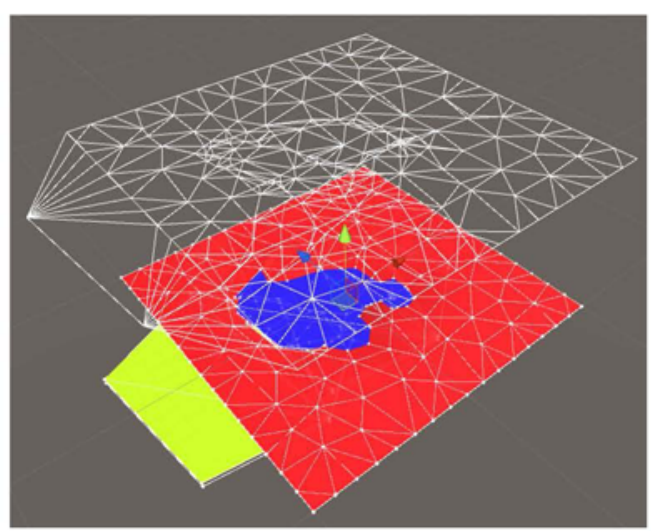

Figure 5. One example of the decision on crossing of the planes in a graphic look.

\section{Conclusion}

It should be noted that in practice this is not all the difficulties that occur when the integration of gaming graphics engines in the unusual structure. However, in spite of all the complexity of the gaming experience in major systems, this appears to be the most useful in the development of modern GIS systems in the coming years.

\section{Reference}

1. GOST P 51794-2008 (Russian Standard) Global navigation satellite systems. Systems of coordinates. Methods of transformations of coordinates of the defined points (2008) 